\title{
Detection of Exoplanets from the Antarctic Plateau
}

\author{
James P. Lloyd \\ Cornell University, Ithaca NY, USA
}

\begin{abstract}
The limitation to ground based astronomy is the Earth's atmosphere. The atmosphere above the Antarctic plateau is fundamentally different in many regards compared to the atmosphere at temperate sites. The extreme altitude, cold and low humidity offer a uniquely transparent atmosphere at many wavelengths. Studies at the South Pole have shown additionally that the turbulence properties of the night time polar atmosphere are unlike any mid latitude sites. The lack of high altitude turbulence combined with low wind speeds presents favorable conditions for interferometry. More recent site testing at Dome $\mathrm{C}$ has revealed the most superb seeing of any site tested. The unique properties of the polar atmosphere can be exploited for Extra-solar Planet studies with differential astrometry, differential phase and nulling interferometers.
\end{abstract}

Keywords. Turbulence, Seeing, Adaptive Optics, Interferometry, Antarctica.

\section{Introduction}

Properties of the atmosphere are the limit for ground based astronomy. Transparency and sky brightness limit the sensitivity of a telescope, and atmospheric turbulence, "seeing", limits resolution. For seeing limited observations, the natural seeing determines the image size, which is of importance not only for resolution, but also for sensitivity as the light from a point source is concentrated into a smaller area, thus suffering less from noise from the contaminating background. For this reason, astronomers have been seeking to deploy telescopes at high altitude sites with stable air for several decades. With the advent of adaptive optics and interferometry, the loss of resolution can be recovered by measuring and tracking the aberrations induced by the atmospheric turbulence. Although it is widely perceived that these techniques can correct for seeing, the seeing enters into the difficulty of the problem in more ways than the image area. The temporal and angular dimensions of coherence of the atmosphere, in addition to the spatial, degrade adaptive optics and interferometry performance. Therefore, these techniques are even more sensitive to degradation by atmospheric turbulence than uncompensated imaging.

It has been recognized for some time that the Antarctic offers the potential for not only exquisitely clear and dark skies, but also for the lowest level of turbulence, with its lowered diurnal variation, high altitude, and remarkably flat topography Gillingham (1993). The combination of superb seeing, low background and high transmission in the thermal infrared allows high levels of performance with an interferometer for the detection and study of extrasolar planets Lloyd et al. (2002), Lloyd et al. (2003a), Lloyd et al. (2003b), Swain et al. (2003a), Swain et al. (2003c), Swain et al. (2003b), Vakili et al. (2005), Vakili et al. (2004), Swain et al. (2004), Fossat et al. (2004).

\section{The Antarctic Atmosphere}

As the optical effect of the turbulence results from the combination of mechanical turbulence with air temperature it is worth examining the processes that generate these. The Palmen-Newton model of the general circulation of the atmosphere (see Figure 1) 


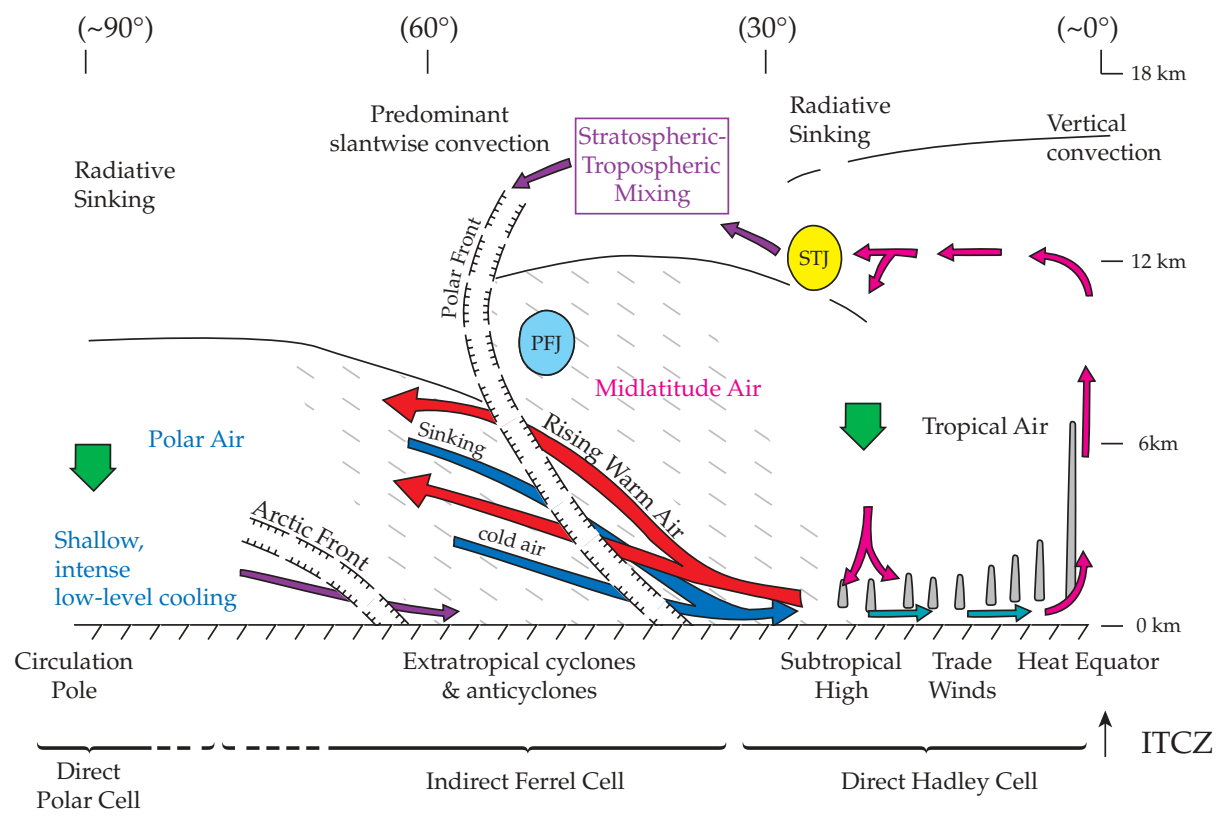

Figure 1. Global circulation and tropopause patterns, adapted from Linacre \& Geerts (1997).

illustrates several of the salient basic processes. The heating of the sun drives convective turnover on large scales in the tropics and mid latitudes. This turnover in turn drives strong winds such as the trade winds at low altitudes and the jetstreams at high altitudes. Variations in local topography, such as mountains, oceans and deserts, result in inhomogeneous heating that constantly produces synoptic weather patterns with large scale inhmogeneous air masses. Of particular concern for astronomy are places where the combination of wind shear and temperature inhomogeneities results in strong turbulence, such as the jet stream and fronts between hot and cold air masses. Periodically, the strong turbulence produced at these interfaces degrades the seeing at mid-latitude sites to very poor levels.

By comparison, the atmosphere over Antarctica is remarkably simple. The sole dominant process is the cooling and sinking of air over the plateau, resulting in katabatic winds. As the Antarctic plateau is nearly uniformly flat and white as a result of the ice cap, there is very little potential to drive convection on large scales. In the wintertime, the atmosphere becomes very nearly adiabatic King \& Turner (1997). In an adiabatic atmosphere, even the injection of mechanical turbulence will not result in optical turbulence, as the density of the air remains uniform at all altitudes. The consequences of these remarkable conditions for the broad context of interferometry and adaptive optics are discussed in Lloyd (2004).

\section{South Pole Seeing}

On the basis of these considerations, the seeing at Antarctic plateau sites should be superior to any mid-latitude sites, as has been recognized previously Gillingham (1993), Gillingham (1991). Comprehensive site testing at the South Pole has characterized not only seeing, but background and transparency at a range of wavelengths Travouillon et al. (2003b), Travouillon et al. (2003a)Travouillon, Ashley, Burton, Storey, Conroy, Hovey, Jarnyk, Sutherland, \& Loewenstein, Travouillon et al. (2002), Storey et al. (2002), Storey et al. (2000), Storey (1998), Storey et al. (1996), Loewenstein et al. (1998). However, the 


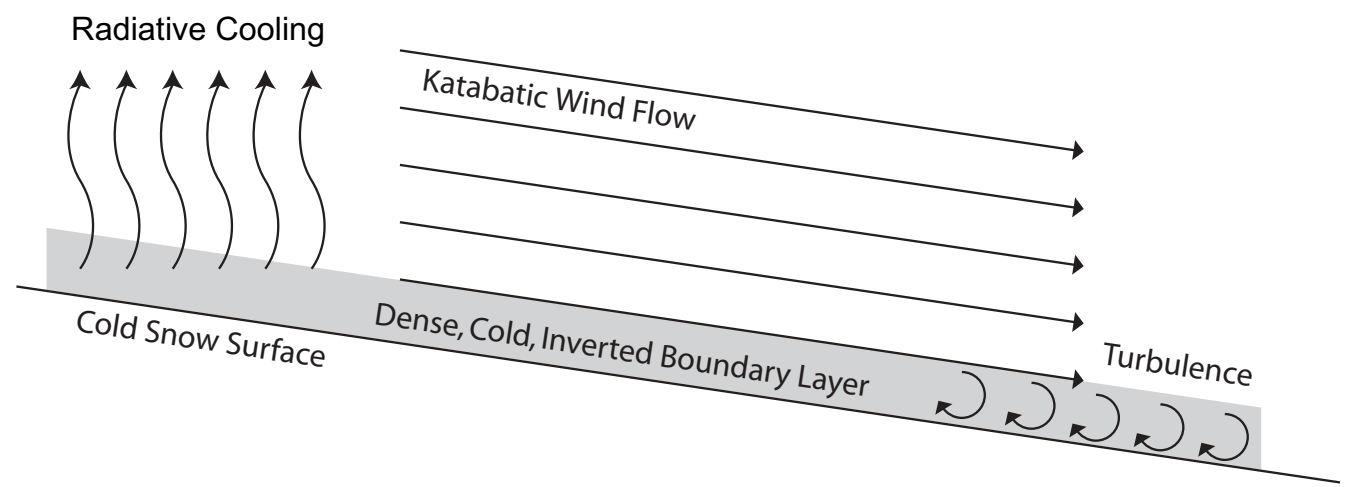

Figure 2. Generation of turbulence in the boundary layer by katabatic wind across the strong wintertime temperature inversion.

seeing at the South Pole was found to be relatively poor, with median seeing of 1.7 " at $0.5 \mu \mathrm{m}$.

The vertical distribution of the turbulence was extensively studied with balloon measurements Marks et al. (1996), Marks et al. (1999), Marks (2002) and SODAR Travouillon et al. (2003b) and found to be confined to a low altitude layer. The radiative cooling of the ice to space results in a strong thermal inversion in the lower 200 meters of the atmosphere. As the South Pole is significantly downhill from the high point of the plateau, it experiences a constant katabatic wind of several meters per second in the wintertime. The sloshing of the cold boundary layer under the action of this wind produces the optical turbulence that results in the relatively poor seeing. It is worth noting, however, that although this seeing is poor for the purposes of image formation, it is less degrading to adaptive optics and interferometry applications due to larger coherence times and isoplanatic angles than comparable seeing at a mid-latitude site Lloyd et al. (2003a). This component of the turbulence, however, is likely to be dramatically lowered in both thickness and strength at the low wind sites on the high plateau. The free atmosphere turbulence measured at the South Pole indicates that under such conditions, exquisite seeing might indeed prevail. Recent observations of the wintertime seeing at Dome C Agabi et al. (2005) indicate that Dome C suffers from a dramatically thinner $(\sim 30 \mathrm{~m})$, but remarkably turbulent boundary layer. Careful study of the boundary layer is clearly required to verity the seeing conditions.

\section{Conclusion}

The ambitious goal of direct detection of exoplanets will clearly require large telescopes and intereferometers at the most superb sites both on the ground and in space. The unique conditions that prevail in the Antarctic atmosphere lead to fundamentally different atmospheric turbulence properties, which are particularly favorable for interferometry and adaptive optics.

\section{Acknowledgements}

This work has been supported in part by the National Science Foundation under grant AST-0335695.

\section{References}

Agabi, A., Aristidi, E., Azouit, M., Fossat, E., Martin, F., Sadibekova, T., Vernin, J., \& Ziad, A. 2005, ArXiv Astrophysics e-prints

Fossat, E., Aristidi, E., \& Agabi, K. 2004, in EAS Publications Series, 125-133 
Gillingham, P. 1993, in Optics in Astronomy: 32nd Herstmonceux Conference, 244+

Gillingham, P. R. 1991, Proceedings of the Astronomical Society of Australia, 9, 55+

King, J. C. \& Turner, J. 1997, Antarctic Meterology and Climatology (Cambridge University Press)

Linacre, E. \& Geerts, B. 1997, Climates and Weather Explained (Routledge)

Lloyd, J. P. 2004, in New Frontiers in Stellar Interferometry, Proceedings of SPIE Volume 5491. Edited by Wesley A. Traub. Bellingham, WA: The International Society for Optical Engineering, 2004., p.190, 190-+

Lloyd, J. P., Lane, B. F., Swain, M. R., Storey, J. W., Travouillon, T., Traub, W. A., \& Walker, C. K. 2003a, in Techniques and Instrumentation for Detection of Exoplanets. Edited by Coulter, Daniel R. Proceedings of the SPIE, Volume 5170, pp. 193-199 (2003)., 193-199

Lloyd, J. P., Oppenheimer, B. R., \& Graham, J. R. 2002, Publications of the Astronomical Society of Australia, 19, 318

Lloyd, J. P., Storey, J. W., Swain, M. R., Traub, W. A., \& Walker, C. K. 2003b, Astronomy in Antarctica, 25th meeting of the IAU, Special Session 2, 18 July, 2003 in Sydney, Australia, 2

Loewenstein, R. F., Bero, C., Lloyd, J. P., Mrozek, F., Bally, J., \& Theil, D. 1998, in ASP Conf. Ser. 141: Astrophysics From Antarctica, 296-+

Marks, R. D. 2002, 385, 328

Marks, R. D., Vernin, J., Azouit, M., Briggs, J. W., Burton, M. G., Ashley, M. C. B., \& Manigault, J. F. 1996, 118, 385

Marks, R. D., Vernin, J., Azouit, M., Manigault, J. F., \& Clevelin, C. 1999, 134, 161

Storey, J., Ashley, M., \& Burton, M. 2002, in ASP Conf. Ser. 266: Astronomical Site Evaluation in the Visible and Radio Range, 524-+

Storey, J. W., Ashley, M. C., \& Burton, M. G. 2000, in Proc. SPIE Vol. 4008, p. 1376-1382, Optical and IR Telescope Instrumentation and Detectors, Masanori Iye; Alan F. Moorwood; Eds., $1376-1382$

Storey, J. W. V. 1998, in ASP Conf. Ser. 141: Astrophysics From Antarctica, 313-+

Storey, J. W. V., Ashley, M. C. B., \& Burton, M. G. 1996, Publications of the Astronomical Society of Australia, 13, 35

Swain, M., Lloyd, J., Traub, W., Walker, C., Stark, A., Lawson, P., Storey, J., Coude du Foresto, V., Fossat, E., Ireland, M., Burrows, A., \& Vakili, F. 2003a, American Astronomical Society Meeting, 203,

Swain, M. R., Coude du Foresto, V., Fossat, E., \& Vakili, F. 2003b, Societa Astronomica Italiana Memorie Supplement, 2, 207

Swain, M. R., Walker, C. K., Storey, J. W., Traub, W. A., \& Lloyd, J. P. 2003c, Astronomy in Antarctica, 25th meeting of the IAU, Special Session 2, 18 July, 2003 in Sydney, Australia, 2

Swain, M. R., Walker, C. K., Traub, W. A., Storey, J. W., Coude Du Foresto, V., Fossat, E., Vakili, F., Stark, A. A., Lloyd, J. P., Lawson, P. R., Burrows, A. S., Ireland, M., MillanGabet, R., van Belle, G. T., Lane, B. F., Vasisht, G., \& Travouillon, T. 2004, in New Frontiers in Stellar Interferometry, Proceedings of SPIE Volume 5491. Edited by Wesley A. Traub. Bellingham, WA: The International Society for Optical Engineering, 2004., p.176, $176-+$

Travouillon, T., Ashley, M. C. B., Burton, M. G., Calisse, P. G., Everett, J. R., Lawrence, J. S., \& Storey, J. W. V. 2002, in SF2A-2002: Semaine de l'Astrophysique Francaise, 19-+

Travouillon, T., Ashley, M. C. B., Burton, M. G., Storey, J. W. V., Conroy, P., Hovey, G., Jarnyk, M., Sutherland, R., \& Loewenstein, R. F. 2003a, 409, 1169

Travouillon, T., Ashley, M. C. B., Burton, M. G., Storey, J. W. V., \& Loewenstein, R. F. 2003b, 400,1163

Vakili, F., Aristidi, E., Schmider, F. X., Jankov, S., Fossat, E., Abe, L., Domiciano, A., Belu, A., Agabi, A., Daban, J.-B., Robbe-Dubois, S., Petrov, R., Vernin, J., Ziad, A., \& Lopez, B. 2005, in EAS Publications Series, 211-217

Vakili, F., Belu, A., Aristidi, E., Fossat, E., Maillard, A., Abe, L., Agabi, K., Vernin, J., Baptiste Daban, J., Hertmanni, W., Schmider, F.-X., Assus, P., Coude Du Foresto, V., \& Swain, M. R. 2004, in New Frontiers in Stellar Interferometry, Proceedings of SPIE Volume 5491. Edited by Wesley A. Traub. Bellingham, WA: The International Society for Optical Engineering, 2004., p.1580, 1580-+ 